\title{
Development and Assessment of Quality Control Phantom for Linearity and Uniformity
}

\author{
Mohamed E. M. Gar-Elnabi' ${ }^{1}$, Wadah M. Ali' ${ }^{2}$, Mohammed A. Ali Omer ${ }^{1,3}$, Adam K. Sam ${ }^{3}$, \\ Ghada A. Edam ${ }^{1}$ \\ ${ }^{1}$ College of Medical Radiologic Science, Sudan University of Science and Technology, Khartoum, Sudan \\ ${ }^{2}$ College of Radiological Science and Nuclear Medicine Technology, The National Ribat University, \\ Khartoum, Sudan \\ ${ }^{3}$ College of Applied Medical Science, Department of Radiology, Qassim University, Buraidah, Saudi Arabia \\ Email: alkajam@gmail.com
}

Received 4 March 2015; accepted 23 May 2015; published 26 May 2015

Copyright (C) 2015 by authors and Scientific Research Publishing Inc. This work is licensed under the Creative Commons Attribution International License (CC BY). http://creativecommons.org/licenses/by/4.0/

\section{(c) (i) Open Access}

\begin{abstract}
Abstract: The aim of this study was to develop a cheap, locally made and friendly applicable phantom for gamma camera quality control and to test its validity relative to standard results (intrinsic and extrinsic spatial linearity and intrinsic and extrinsic uniformity) of gamma camera SPECT. And the significance of this experimental study was to introduce a multi-purpose phantom for gamma camera which could overcome the risk accompanied by quality control test procedure such as detector crystal damage and the appearance of moiré patterns. The results of the developed phantom showed an average count difference of $0.7 \%$ relative to the standard phantom and about $4 \%$ in $\mathrm{X}$ - to $\mathrm{Y}$-axis directions relative to the standard phantom. Also, the measured absolute linearity was $0.63 \mathrm{~mm}$ in $X$ direction and $0.64 \mathrm{~mm}$ in $Y$ direction for the UFOV compare with 0.70 $\mathrm{mm}$ value of acceptance test. And the I.U. and D.U. of the developed phantom were $3.18 \%$ and $2.27 \%$ respectively for the UFOV relative to the standard phantom I.U. and D.U. (2.0\% and $1.5 \%)$ for the UFOV respectively.
\end{abstract}

\section{Keywords}

Gamma, Camera, Phantom, Flood-Field, Uniformity, Linearity, Quality Control

\section{Introduction}

Since 1958, Anger gamma camera system has been introduced to the field of nuclear medicine studies [1]. Many

How to cite this paper: Gar-Elnabi, M.E.M., Ali, W.M., Omer, M.A.A., Sam, A.K. and Edam, G.A. (2015) Development and Assessment of Quality Control Phantom for Linearity and Uniformity. Open Journal of Radiology, 5, 59-65. 
efforts and innovations have contributed significantly to the recent status of radiology and nuclear medicine technology; represented in computed tomography (CT), digital radiography, diagnostic ultrasound, fundamentals of nuclear magnetic resonance imaging (MRI)) and (single-photon emission computed tomography [SPECT] and position emission tomography [PET]) in the years of 1960s and 1970s [2]. Such technique actually measures the coincidence of the two annihilated photons that follow positron decay in the organ of interest to diagnose pathologic evidence, e.g. studies of dementia and/or epileptic foci, and in the determination of myocardial viability. The technique is also indicated in the study of various malignancies, including staging, therapy response and follow-up [2]. The development of gamma camera SPECT has been accompanied with regular developing in the field of computers programs and quality assurance technologies to optimize the SPECT performance and eliminating the errors, as SPECT has well-recognized limitations in spatial resolution and statistical quality [3] [4].

The performance parameter most commonly evaluated as a part of a routine gamma camera quality control program (QCP) includes uniformity, spatial resolution, spatial linearity, and energy resolution and peaking [5]. Nuclear medicine is critically dependent on the accurate and reproducible performance of clinical radionuclide counting and imaging instrumentation. Quality control may be defined as an established set of ongoing measurements and analysis designed to ensure that the performance of a procedure or instrument is within a predefined acceptable range, which is thus a critical component of routine nuclear medicine practice [6] [7], and routine quality assurance program should be developed and followed where tests are performed on regular interval [8]. A general phantom used for quality assurance can be expensive and not always easily accessible. In addition, due to the fact that there are different camera configurations and detector sizes, such phantoms should, in some cases, be camera specific. It will therefore be of great benefit to nuclear medicine departments to have an alternative and cheaper way to manufacture phantoms according to their own need [8]. Also in some QA procedure such as spatial linearity in which the phantom placed directly over the detector with or without collimator relative to $\mathrm{X}$ and $\mathrm{Y}$ coordinators; the visual inspection of the obtained image should show straight bars in both coordinates as has been mentioned by Pat [5], with consideration to the risk of crystal breaking attained with collimator removal (intrinsic test) or moiré patterns appearance due to interplay of the bar or hole pattern and the lead septa of the collimator (extrinsic test) [9]. System linearity is more sensitive to change in PMT performance; fortunately, such change is also manifested as a change in system uniformity. The 4-quadrant bar phantom is commonly used to measure system resolution, while it is not ideally suiting to measure system linearity as orthogonal hole pattern or the parallel line equal spacing phantom does, where they also allow assessment of both linearity and resolution [10]. The flood field uniformity (intrinsically without collimator or extrinsically with collimator) of a scintillation camera is the ability of the camera to produce a uniform image when exposed to a homogeneous spatial distribution of gamma rays [11] [12]; such parameters have validity range, for differential uniformity and integral uniformity are $(1.0 \%-2.5 \%)$ and $(1.5 \%-3.5 \%$ or $2 \%-4 \%)$ respectively [3] [13]. Hence, when the differential uniformity exceeds $3 \%$, the maintenance service should be carried out on the gamma camera [3] [14]. For the potential limitation of the QA phantom highlighted above, the designing of specific phantom became evitable.

\section{Method}

The designed phantom has been excerpted from the phantom parameters stated by International Atomic Energy Agency DOC-602 [15], NEMA-2001 [16], Ng et al. [17], Holstensson et al. [18] [19] and Islamian et al. [20], which consists of four quadrate bars as recommended by Zanzorico et al. [5].

The frontal part of the phantom made of Perspex $(42 \times 42 \times 10 \mathrm{~cm})$ shown in Figure 1 that simulates the four quadrant bars phantom each one was $20 \times 20 \mathrm{~cm}$, which have been grooved by laser cutting bed (BCL-B series model BCL1318B china 1991). The first quadrant implies 26 grooves with dimensions of $18 \times 0.35 \mathrm{~cm}$ and separated from each other by distance of $3.5 \mathrm{~mm}$. The second quadrant contains 30 grooves as $18 \mathrm{~cm} \times 3 \mathrm{~mm}$ and each adjacent grooves was separated by distance of $3 \mathrm{~mm}$, the third quadrant contains 32 grooves with dimension of $18 \mathrm{~cm} \times 0.25 \mathrm{~cm}$ separated by $2.5 \mathrm{~mm}$ distance, and the forth quadrant contains 32 Grooves with $18 \mathrm{~cm} \times$ $2 \mathrm{~mm}$ and separated from each other by factor of $2 \mathrm{~mm}$. The edge of the phantom, i.e. the remaining $2 \mathrm{~cm}$; a big grooves was made with dimension of $36 \mathrm{~cm}$ length and $5 \mathrm{~mm}$ width, which is used to measure the linearity of the gamma camera by measuring Modulation transfer function (MTF) of the Line Spread Function (LSF). The back part simulates the liquid flood phantom made of Perspex $(42 \times 42 \times 1 \mathrm{~cm})$ (Figure 2) and has orifice $0.5 \mathrm{~cm}$ to be fill with a liquid radioactive material, controlling the air bubbles and insuring the homogeneity. Then some 


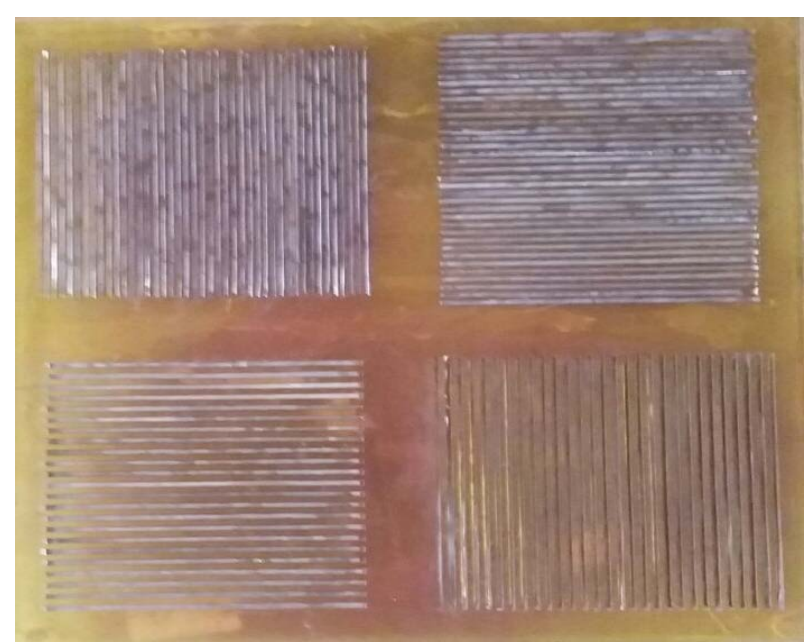

Figure 1. The frontal part of the phantom made of Perspex $(42 \times 42 \times 10 \mathrm{~cm})$ that simulates the four quadrant bars phantom each one was $20 \times 20 \mathrm{~cm}$.

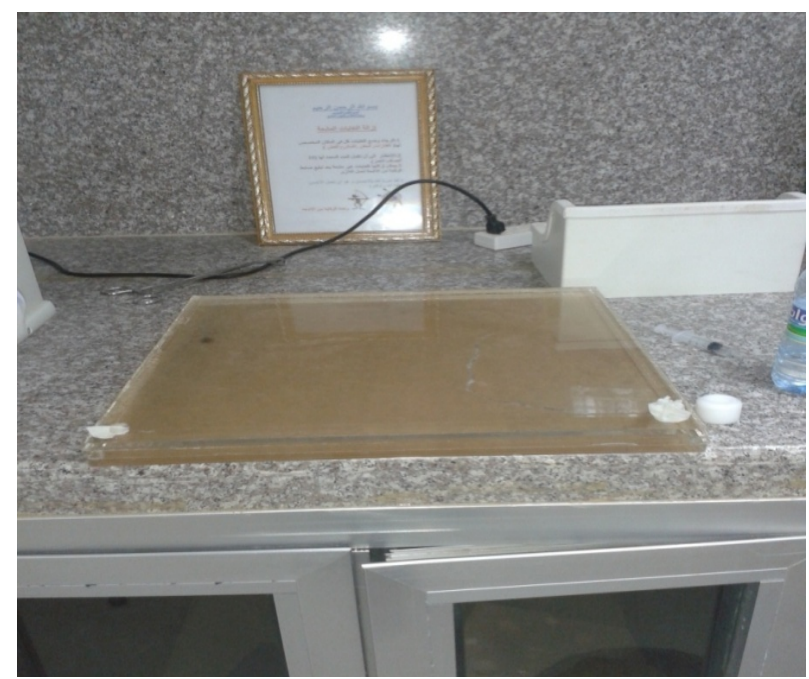

Figure 2. The back part simulates the liquid flood phantom made of Perspex $(42 \times 42 \times 1 \mathrm{~cm})$ and has orifice $0.5 \mathrm{~cm}$ to be fill with a liquid radioactive material.

lead wires have been fabricated in smooth and fine shapes according the dimension of the grooves $(18 \times 0.35 \mathrm{~cm}$, $18 \times 0.3 \mathrm{~cm}, 18 \times 0.25 \mathrm{~cm}, 18 \times 0.2 \mathrm{~cm}$ and $36 \times 0.5 \mathrm{~cm}$ ) which then have been mounted in the relevant grooves in the front quadrants. Then a mixture of water $(1500 \mathrm{ml})$ and $\mathrm{Na} 99 \mathrm{mTcO} 4(1.3 \mathrm{mCi})$ has been flushed into the phantom via the orifice, shacked to maintain the homogeneity and air bubbles free. Then the slit lead phantom centered on the liquid flood source $(1.3 \mathrm{mCi} 99 \mathrm{mTc})$ phantom (Figure 3$)$ and both have been placed at the floor five times the FOV distance, and such assemble has been centered to the head of gamma camera for measuring the flood field uniformity and linearity with a usage of full detector mask, 0 degree orientation, Low Energy High Resolution collimator, Intrinsic flood correction, $140 \mathrm{KeV}$ energy peak and $20 \%$ energy window width. Then serial sets of images was acquired using one variable parameter of matrix size of the image which include image matrix range from $64 \times 64 \times 8$ to $1024 \times 1024$ with 16 or 32 word.

\section{Results}

The following results shows the data of Gama Camera SPECT as counts obtained by the developed phantom in 
$\mathrm{X}$-axis and $\mathrm{Y}$-axis direction for assessing the linearity as well as the flood field uniformity obtain from the gamma camera and the analyzed one using IDL program which is constructed as phantom specific program to convert the flood field uniformity of the gamma camera into point spread function image.

\section{Discussion}

Linearity in Figure 4 shows the counts (dps) distributions spectrum in X-axis and Y-axis direction via phantom lead slits. In which there is semi symmetrical and superimposition between the spectrums obtained in $\mathrm{X}$-axis and that in $\mathrm{Y}$-axis. The average count has been compared with that obtained by standard phantom which gives an acceptable variation of $0.7 \%$ indicating there is acceptable linearity between the developed phantom and the standard one. The difference in counts between $\mathrm{X}$-axis and $\mathrm{Y}$-axis direction has been plotted versus phantom slits (Figure 5), which in turn reveals that: there is about $4 \%$ difference per each slit of phantom relative to standard one as shown in Figure 5. Also the absolute linearity has been measured for the central field of view (CFOV) of the gamma camera depending on NEMA specifications with respect to the real position coordinate $\mathrm{x}$

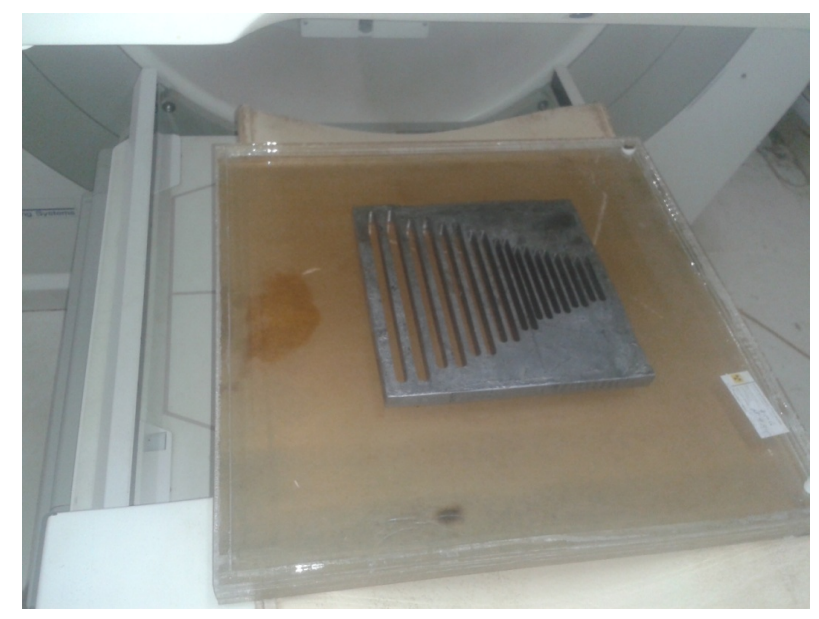

Figure 3. The slit lead phantom centered on the liquid flood source $(1.3 \mathrm{mCi} 99 \mathrm{mTc})$ phantom.

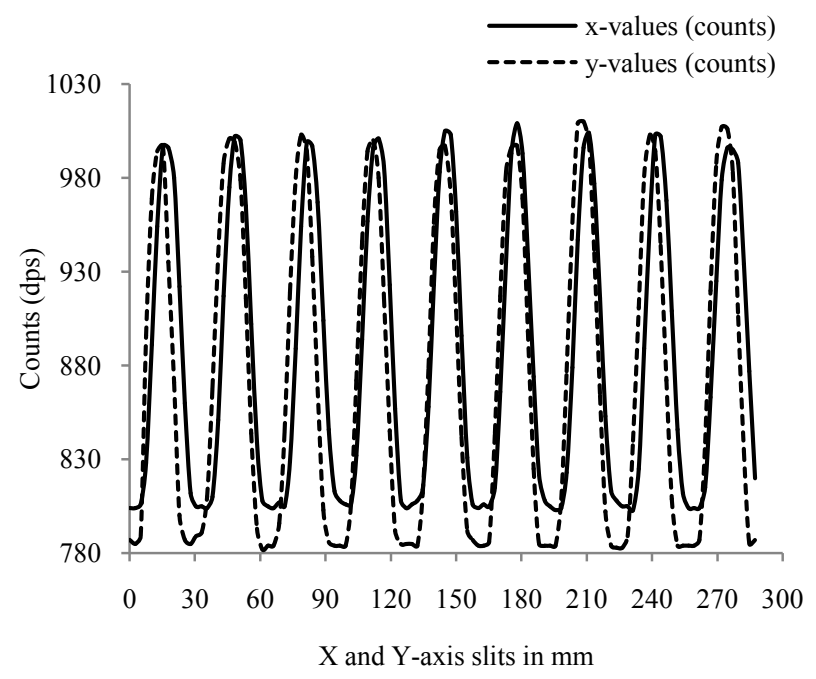

Figure 4. The counts distributions in $\mathrm{X}$ and $\mathrm{Y}$-axis direction via phantom lead slits (the counts refers to the intensity of photons come out between the spaces of lead strip when the phantom aligned parallel to $\mathrm{X}$-axis and $\mathrm{Y}$-axis direction). 
and $\mathrm{y}$ which shows the counts (dps) versus phantom slits. And the calculation has been carried out using line spread function (LSF) which in turn determined as the average of the interpolated half maximum locations on both sides of each peak. The value of spatial linearity is calculated as the standard deviation of the location of the peaks in each slit and was $0.631 \mathrm{~mm}$ in X-axis direction and $0.636 \mathrm{~mm}$ in Y-axis direction for the useful field of view (UFOV) with no significant deviations between the $\mathrm{X}$-axis and $\mathrm{Y}$-axis direction. The comparison this result relative the acceptance test of the gamma camera and the recommendation values from manufacture, the researchers found that there is no significant difference as the acceptance values for absolute linearity for UFOV is $0.70 \mathrm{~mm}$ while the manufacture recommended a value of $0.28 \mathrm{~mm}$.

Uniformity: Two uniformity parameters as integral and differential (I.U. \& D.U.) were measured and evaluated using interactive data language (IDL) program. The I.U focused on the whole numbers of the pixels that contain the image while the D.U. measured for every $5 \times 5$ rows and column of the pixels. Serial of images were taken using the low-cost locally manufactured phantom according to the NEMA standard publication [16] in acquisitions of the image and analysis. Figure 6 shows the image uniformity of the developed flood phantom obtained from gamma camera SPECT which is processed by using IDL program and shown in Figure 7 which is a point spread function spectrum with homogeneous surface. The analysis revealed that the I.U. and D.U. were $3.18 \%$ and $2.27 \%$ for UFOV respectively. And in comparison with the standard acceptance tests I.U. and D.U. for the UFOV which are $2.0 \%$ and $1.5 \%$ respectively, the analysis deduced that: there is no significant difference; hence the phantom could be applicable for flood field uniformity measurement.

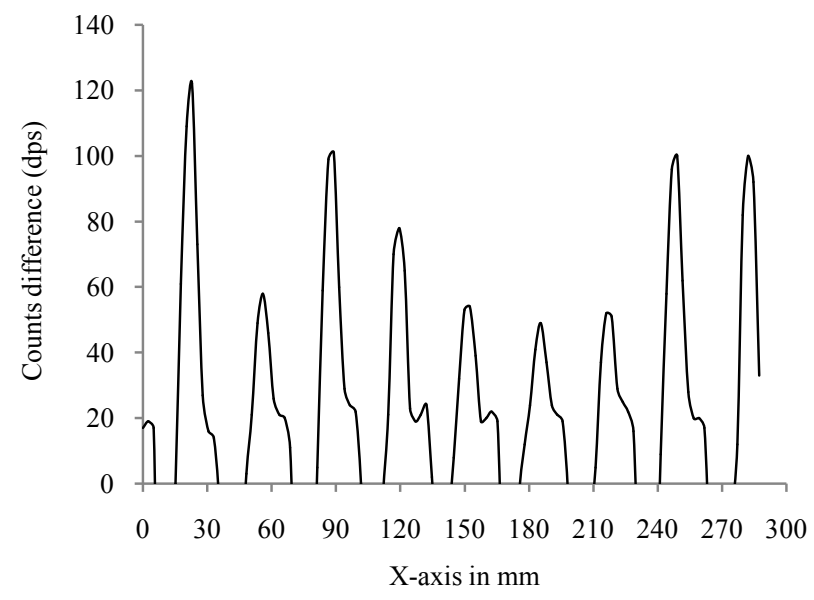

Figure 5. The counts difference in $\mathrm{X}$ - and $\mathrm{Y}$-axis direction via phantom lead slits which in turn indicating the difference in photon intensity in $\mathrm{X}$ - and $\mathrm{Y}$-axis direction.

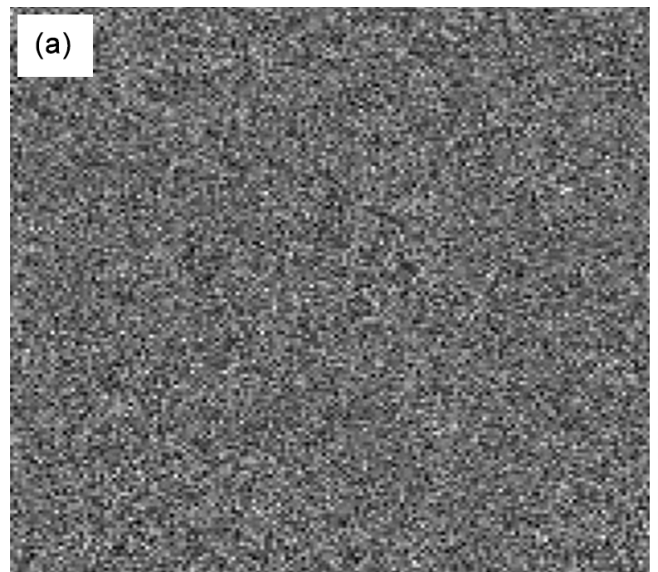

\section{(b)}

Figure 6. (a) The image uniformity of the developed flood phantom obtained from gamma camera SPECT and (b) for the standard phantom. 


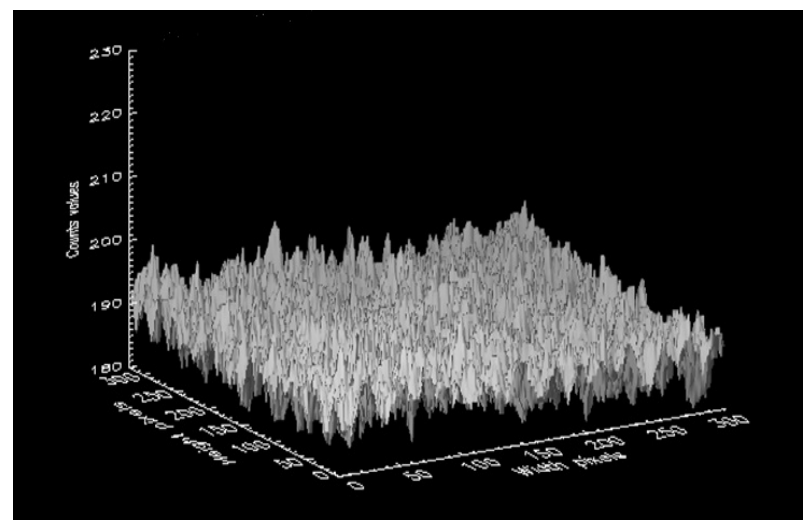

Figure 7. The point spread function of flood field uniformity obtained by developed phantom and analyzed by IDL program (X, $\mathrm{Y}$ and $\mathrm{Z}$ are the pixel width, pixel length and the counts respectively).

\section{Conclusion}

The developed phantom could be used with acceptable accuracy in the field of nuclear medicine to carry out the quality control tests of gamma camera SPECT, which was considered as a general multi-functional phantom as it could measure the linearity and flood field uniformity as well as the resolution.

\section{References}

[1] Anger, H.O. (1958) Scintillation Camera. Review of Scientific Instruments, 29, 27-33. http://dx.doi.org/10.1063/1.1715998

[2] Seo, Y., Aparici, C.M. and Hasegawa, B.H. (2008) Technological Development and Advances in Single-Photon Emission Computed Tomography/Computed Tomography. Seminars in Nuclear Medicine, 38, 177-198. http://dx.doi.org/10.1053/j.semnuclmed.2008.01.001

[3] Cherry, S.R., Sorenson, J.A. and Phelps, M.E. (2003) Physics in Nuclear Medicine. Saunders, Philadelphia.

[4] Jaszczak, R.J., Coleman, R.E. and Lim CB. (1980) SPECT: Single Photon Emission Computed Tomography. IEEE Transactions on Nuclear Science, NS-27, 1137-1153.

[5] Pat, Z. (2008) Routine Quality Control of Clinical Nuclear Medicine Instrumentation: A Brief Review. The Journal of Nuclear Medicine, 49, 1114-1131. http://dx.doi.org/10.2967/jnumed.107.050203

[6] Lin, P.-J. P., Borras, C., et al. (1993) Specification and Acceptance Testing of Computed Tomography Scanner. American Association of Physics in Medicine, New York.

[7] Graham, L.S., Fahey, F.H., Madsen, M.T., van Aswegen, A. and Yester, M.V. (1995) Quantitation of SPECT Performance: Report of Task Group 4, Nuclear Medicine Committee. Medical Physics, 22, 401-409. http://dx.doi.org/10.1118/1.597605

[8] Van Staden, J.A., Dv Raan, H., lotter, M.G., Van Aswegan, A. and Herbst, C.P. (2007) Production of Radioactive Quality Assurance Phantoms Using Standard Inkjet Printer. Physics in Medicine and Biology, 52, N 329-N 337.

[9] Bone, F.J., Graham, K.D. and Dowdey, J.E. (1971) Image Aberration Produced By Multi-Channel Collimators for a Scintillation Camera. Radiology, 98, 329-334. http://dx.doi.org/10.1148/98.2.329

[10] O’Conner, M.K., Clinic, M. and Rochester, M.N. Quality Control of Scintillation Cameras (Planar and SPECT).

[11] Doed, S.B. (1994) Practical Radiation Protection and Applied Radiobiology. W.B. Saunders Co., Philadelphia.

[12] Smith, E.M. (1998) Scintillation Camera Quality Control, Part 1: Establishing the Quality Control Program. Journal of Nuclear Medicine Technology, 26, 9-13.

[13] Ejeh, J.E., Adedapo, K.S., Akinlade, B.I. and Osifo, B.O. (2011) Gamma Camera Intrinsic Uniformity in an Unstable Power Supply Environment. Hellenic Journal of Nuclear Medicine, 14, 146-148.

[14] Young, K.C., Kouris, K., Awdeh, M. and Abdel-Dayem, H.M. (1990) Reproducibility and Action Level for Gamma Camera Uniformity. Nuclear Medicine Communications, 11, 95-101. http://dx.doi.org/10.1097/00006231-199002000-00003 
[15] International Atomic Energy Agency (1991) Quality Control of Nuclear Medicine Instrumentations. IAEATECDOC602.

[16] National Electrical Manufacturers Association (NEMA) (2001) Performance Measurements of Scintillation Cameras. NEMA Standards Publication NU 1-2001, Global Engineering Documents. Washington DC.

[17] Ng, A.H., Ng, K.H., Dharmendra, H. and Perkins, A.C. (2009) A Low-Cost Phantom for Simple Routine Testing of Single Photon Emission Computed Tomography (SPECT) Cameras. Applied Radiation and Isotopes, 67, 1864-1868. http://dx.doi.org/10.1016/j.apradiso.2008.10.010

[18] Holstensson, M., Partridge, M., Buckley, S.E. and Flux, G.D. (2010) The Effect of Energy and Source Location on Gamma Camera Intrinsic and Extrinsic Spatial Resolution: An Experimental and Monte Carlo Study. Physics in Medicine and Biology, 55, 1735-1751. http://dx.doi.org/10.1088/0031-9155/55/6/013

[19] Holstensson, M., Hindrof, C., Ljungberg, M., Partridge, M. and Flux, G.D. (2007) Optimization of Energy-Window Setting for Scatter Correction in Quantitative ${ }^{111}$ in Imaging: Comparison of Measurements and Monte Carlo Simulations. Cancer Biotherapy and Radiopharmaceuticals, 22, 136-142. http://dx.doi.org/10.1089/cbr.2007.307

[20] Islamian, J.P., Toossi, M.T.B., Momennezhad, M., Naseri, S. and Ljungberg, M. (2012) Simulation of a Quality Control Jaszczak Phantom with SIMIND Monte Carlo and Adding the Phantom as an Accessory to the Program. Iranian Journal of Medical Physics, 9, 135-140. 